\title{
tic\&société
}

Vol. $1, n^{\circ} 1 \mid 2007$

De TIS à tic\&société : dix ans après

\section{Déstructuration et restructuration du journalisme}

\section{François DEMERS}

\section{OpenEdition}

\section{Journals}

Édition électronique

URL : http://journals.openedition.org/ticetsociete/298

DOI : 10.4000/ticetsociete.298

Éditeur

Association ARTIC

Référence électronique

François DEMERS, « Déstructuration et restructuration du journalisme », tic\&société [En ligne], Vol. 1, n

${ }^{\circ} 1$ | 2007, mis en ligne le 20 novembre 2009, consulté le 19 avril 2019. URL : http://

journals.openedition.org/ticetsociete/298; DOI : 10.4000/ticetsociete.298 


\section{Déstructuration et restructuration du journalisme}

François DEMERS, Professeur

Faculté des Lettres

Université Laval

Adresse postale :

Pavillon Louis-Jacques- Casault, bureau 5413,

Faculté des Lettres, Université Laval

Québec, Canada

G1K 7P4

Adresse de courriel :

Francois.Demers@com.ulaval.ca

François DEMERS, Ph.D. en science politique, est professeur titulaire au Département d'information et de communication de l'Université Laval (ville de Québec) où il enseigne depuis 1980. Auparavant, il avait été journaliste professionnel pendant 15 ans. II a créé un cours à distance totalement sur Internet portant sur le journalisme en ligne. II est membre de l'équipe de recherche : Pratiques novatrices en communication publique (PNCP).

http://www.com.ulaval.ca/personnel/professeurs/francois_demers/index.php 


\title{
François DEMERS
}

\section{Résumé}

Les «nouvelles » technologies contribuent, en matière de journalisme, à désunir ce qui avait été assemblé et qui constituait jusqu'à récemment les formules gagnantes. En même temps, les acteurs tâtonnent, innovent et recherchent de nouveaux assemblages capables de durer. Ce texte présente trois déstructurations-restructurations devenues visibles au cours de la récente décennie. La première se produit au niveau des modèles d'affaires où émergent les télévisions transnationales en information continue, les quotidiens gratuits et l'Internet. La seconde réorganise les contenus des médias généralistes. D’un côté, publicité, divertissement et information se dissocient et se donnent des médias spécialisés; de l'autre, l'hybridation s'accélère quand les trois (3) continuent de concert dans les mêmes supports. La troisième restructuration cible l'information elle-même dont les composantes se dissocient: la production de l'actualité demeure entre les mains des professionnels pendant que la fonction éditoriale (les commentaires) se répand du côté du « journalisme citoyen » sur Internet et que le magistère journalistique se replie sur la création d'images de journalistes-vedettes et de marques de commerce.

Mots-clés : journalisme, information, actualité, éditorial, magistère

\begin{abstract}
In the field of journalism, " new » technologies are helping to dissolve what have long been considered winning formulas. At the same time, and with the help of the same technologies, the interested parties experiment and innovate as they seek new, successful mixes. This text presents three such cases of destructuration- restructuration that have affected journalism in the last decade. The first one concerns new business models that have given rise to transnational all-news television stations, free dailies and Internet. The second involves the reorganization of the content of generalist (catch-all) medias. On one side, publicity, entertainment and information are separated and give rise to distinct media; on the other, hybridation intensifies when they remain together in the same supports. The third restructuration goes to the very heart of journalistic information itself with the dissociation of its various components. : news production (newsmaking) remains in the hands of professional journalists while the editorial function (op-ed) is dispersed through so-called « citizen journalism » on the Internet and journalistic authority restricts itself to creating images of star journalists and commercial brands.
\end{abstract}

Keywords : Journalism, information, newsmaking, op-ed, authoritative posture 


\section{Resumen}

En el campo del periodismo, las « nuevas » tecnologías contribuyen a separar aquello que habia sido juntado y que hasta el momento se presentaba como formula exitosa. Al mismo tiempo, los actores, con la ayuda de las mismas tecnologías, tantean, innovan y buscan nuevos ensamblajes. Este texto presenta tres casos de desestructuraciónrestructuración en la actividad periodistica de la ultima década. La primera se produce en el nivel de los modelos de negocio que se sitúan en la base de la emergencia de las cadenas de televisión de información continua, los diarios gratuitos e Internet. La segunda, reorganiza los contenidos de los medios generalistas. Por un lado, la publicidad, el entretenimiento y la información se disocian y se convierten en medios especializados; por otro lado, se hibridan cuando los tres (3) coexisten en los mismos soportes. La tercera restructuración apunta a la propia información periodistica: la producción de la actualidad (las noticias) queda en los manos de los profesionales mientras que la función editorial (los comentarios) se relacionan con el «periodismo ciudadano » en Internet; a la vez se asiste a un magisterio periodístico basado en la creación de periodistas-estrellas y de marcas de comercio

Palabras clave : periodismo, información, actualidad, editorial, magisterio 


\section{François DEMERS}

Cet article reprend la piste ouverte en 1996 dans la revue sur support papier Technologies de l'information et Sociétés (TIS) par un premier texte portant la même thématique (Demers, 1996). II soutient que les nouvelles technologies contribuent, en matière de journalisme, à désunir ce qui avait été assemblé et qui constituait les formules qui se sont révélées gagnantes du milieu du XIXe siècle à la fin du $X_{X e}{ }^{1}$. En même temps, poussés par les changements techniques, les acteurs tâtonnent, innovent et recherchent de nouvelles combinaisons d'éléments capables de durer. Ainsi, déstructuration et restructuration ${ }^{2}$ vont de pair.

Cette grille de lecture s'appuie sur trois autres propositions antérieures qui sont prises comme axiomes dans le cadre de ce texte. La première rappelle que la notion de journalisme, dans la culture d'une société occidentale développée comme le Canada, quoique entourée de flou (Ruellan, 1993), désigne une catégorie spécifique de contenu des médias de masse (souvent nommée " information »), une activité professionnelle particulière liée à ce contenu ainsi qu'un groupe spécifique de travailleurs : les journalistes. L'important aussi, c'est que ce journalisme ne vient pas seul, il est spontanément posé comme partie d'un ensemble : les médias. La deuxième proposition prolonge la première en ce qu'elle situe les technologies parmi les composantes de l'ensemble dans lequel s'inscrit le journalisme, à la manière de Jean de Bonville (1988) quand il raconte la naissance du journalisme d'information au Canada. Si la présente analyse met l'accent sur les changements techniques, ce n'est pas parce qu'ils sont posés comme LE facteur moteur du changement. La causalité demeure en effet ouverte à un examen empirique qui devrait d'ailleurs être réalisé dans l'éclairage des interactions de la technique, pas à pas, avec les autres facteurs (politiques, culturels, économiques) de même qu'avec les rapports de pouvoir et d'intérêts entre les acteurs sociaux. Cette approche mise de l'avant par I'historien James Carey (Attallah, 1989), fonde d'ailleurs le troisième postulat préalable : les changements technologiques qui occupent le devant de la scène actuelle font partie d'une " filière ", concept aussi développé par Carey, celle de la numérisation des informations (le langage $0 / 1$ des ordinateurs) qui se déploie depuis le milieu du siècle dernier dans tous les secteurs d'activité, et dont toutes les possibilités d'innovation n'ont sans doute pas encore été épuisées.

Le cadre d'analyse cherche donc à lire le journalisme d'aujourd'hui comme une transition, le moment de la fin d'une configuration (jeu de règles connues et

\footnotetext{
${ }^{1}$ Gagnant veut dire ici : rentable financièrement et valorisée par une majorité d'acteurs.

2 Structure désigne ici : ordre interne organisé et stable des éléments d'un ensemble formant un tout.
} 


\section{Déstructuration et restructuration du journalisme}

stables) à la naissance d'une nouvelle ${ }^{3}$. Au cœur de la configuration sortante : les grands magazines, quotidiens et chaînes de télévision pionnières, souvent nommés: médias traditionnels ou médias généralistes ${ }^{4}$. Les combinaisons d'éléments que ces médias avaient unis en des ensembles intégrés, stables et fonctionnels, sont toujours présents dans les esprits de plusieurs; leur stabilité (relative) dans le temps les avaient rendus familiers, sinon naturalisés. Aussi, la dissociation de leurs éléments, en cours de manière de plus en plus accélérée depuis les années 1980, bloque souvent la pensée sur ce qui se défait, comme un regard nostalgique dans le rétroviseur, plutôt que sur les nouvelles combinaisons en émergence. II conviendra donc ici, comme dans le texte de 1997, de rappeler la configuration dans laquelle s'insérait le journalisme dans les médias traditionnels, mais pour mieux faire ressortir quelques nouvelles combinaisons où le journalisme est présent.

Le premier moment de l'exposé qui va suivre pose que la déstructuration des formules traditionnelles se produit au niveau des modèles d'affaires qui ont permis au journalisme, comme pratique et produit, de s'inscrire dans des activités commerciales et industrielles lucratives. Le second, restreignant l'angle d'examen, rappelle que l'information journalistique n'est qu'un des contenus, un des ingrédients de la formule globale des médias traditionnels caractérisés par le projet de rejoindre tout le public d'un territoire, à la manière du supermarché en position de monopole. La troisième partie réduit à nouveau le propos et attire l'attention sur le fait que l'information journalistique est un composite d'au moins trois activités distinctes qui ont elles aussi tendance à se dissocier. Dans l'ensemble, les chapitres jouent le rôle d'exemples de la fécondité de la proposition de départ : les morceaux composant les formules gagnantes d'hier,

\footnotetext{
${ }^{3}$ Configuration au sens du sociologue anglo-allemand Norbert Elias qui propose de voir le jeu social autour d'un enjeu, dans ce cas-ci : le journalisme, comme un ensemble de règles d'interrelation entre les acteurs intéressés. Le terme configuration met l'accent sur la stabilité des règles du jeu (Elias, 1981). La question devient alors de déterminer comment se font les changements de configuration, que l'on doit considérer comme des ruptures de grande ampleur.

${ }^{4}$ Ils sont nommés «traditionnels " par rapport aux nouvelles formes de médias tels les quotidiens gratuits dans les transports en commun ou certains sites Internet, si ce n'est l'Internet lui-même considéré comme un média. On parle aussi d'eux comme des médias "généralistes " parce qu'offrant à la fois un éventail de produits couvrant la gamme complète de la communication de masse : information, publicité et divertissement, et un menu thématique cherchant à répondre à toutes les attentes: sports, documentaires, films, jeux, débats politiques, etc. Ce texte utilisera indifféremment l'une et l'autre expression pour les désigner.
} 


\section{François DEMERS}

en raison notamment des possibles ouverts par les nouvelles technologies, se séparent au profit de nouvelles combinaisons d'ingrédients dans une course générale vers de nouveaux ensembles gagnants stables. Enfin, une fenêtre sera ouverte sur certains défis que ce processus entraîne pour les journalistes professionnels, employés et salariés des médias.

\section{La formule généraliste}

Ce qui est ici posé comme en voie de déconstruction est un ensemble historiquement construit. Diverses études rappellent en effet que les entreprises d'information qui ont émergé au XIXe siècle dans les pays développés occidentaux, ont combiné pratiques antérieures, innovations techniques et culture de la Modernité, pour devenir commerces. Ils ont emprunté, bricolé, innové, pour accoucher finalement d'un produit industriel et commercial unique. Ils ont eu besoin d'innovations techniques: la machine à vapeur, les chemins de fer, les tramways, le papier de cellulose de bois, etc. pour industrialiser leur production. Ils ont appliqué les méthodes de production en série du fordisme pour assurer la production quotidienne d'un journal de plus en plus épais. En tant que pratique discursive, ils ont prolongé et intégré les pratiques d'expression et de polémique politiques des feuilles d'opinion antérieures. Ils ont inventé la publicité comme espace promotionnel payé par les commerçants et contrôlé par eux. Ils ont créé la figure du reporter sur le modèle de la caméra et en ont fait un ouvrier interchangeable en raison de sa formation à l'objectivité du positivisme. Finalement, ils ont privilégié l'événement dans sa forme «nouvelle», comme locomotive de la vente des exemplaires. (De Bonville, 1988)

Les infrastructures techniques porteuses des contenus vers les consommateurs ont continué par la suite à se déployer, selon leur filière, sans que la «structure » de la formule d'ensemble change sensiblement. Par exemple, en télévision, le marigage s'est fait au départ avec les ondes hertziennes. Cette alliance-là est moribonde. Il y a eu ensuite l'usage des câbles en cuivre, et la fibre optique. L'innovation a fait la bonne fortune des câblodistributeurs et c'est le mariage encore dominant actuellement au Canada. Plus récemment, le satellite est venu disputer le terrain au câble. Présentement, c'est la télévision sur Internet qui monte, ce support se convertissant rapidement aux contenus vidéos, au fur et à mesure que des équipements performants deviennent d'usage courant ${ }^{5}$.

\footnotetext{
${ }^{5}$ Le temps (30-10-2006). MULTIMÉDIA : Dans l'ombre de YouTube, des centaines de chaînes de télévision apparaissent. Hollywood s'inquiète, par Michael Lew. Quotidien suisse en ligne. <http://www.le temps.ch/template/print.asp?article=192715>.
} 


\section{Déstructuration et restructuration du journalisme}

Mais pendant tout ce temps, la formule dominante est restée la « généraliste » visant à se faire tout à tous : en offrir pour tous les goûts et, par là, rejoindre toute l'audience possible. Avec une formule d'affaires à l'avenant: conquérir le monopole sur un marché géographique donné.

\subsection{Le dé-montage financier}

Aujourd'hui, une seule chose paraît certaine quand on considère le panorama global, c'est le recul des médias généralistes dans un environnement où se multiplient les médias spécialisés. La chute des tirages des quotidiens traditionnels ${ }^{6}$ et la perte d'audience des télévisions pionnières sont en effet devenues une donnée centrale de la réflexion sur le changement des médias et du journalisme. Des experts spéculent sur les causes et sur les rétablissements possibles. Les colloques se multiplient. Celui qui a été tenu à Montréal le 24 novembre 2006, sous le thème: Radiodiffusion et nouveaux médias : modèles d'affaires et régulation aura notamment permis de rassembler des données empiriques très abondantes sur l'état de la question, sur les angoisses, les expériences et les formules à l'essai un peu partout dans les pays développés, là où la déstructuration / restructuration est la plus frappante ${ }^{7}$. Du portrait d'ensemble, on peut dégager un certain nombre de tendances qui vont dans le sens de la proposition guide de ce texte. On y voit par exemple des contenus écrits qui paraissaient rivés au support papier, être déroulés sur les écrans des ordinateurs, des téléviseurs et même des téléphones. Les images animées,

Le Devoir (03-02-2007). 2007 sera l'année de la vidéo en ligne au Québec, par Bruno Guglielminetti. Mercredi, p. B7. L'argument y porte sur les sites de vidéos amateurs tels YouTube, Myspace (le site le plus visité aux Etats-Unis), en France: Dailymotion, Skyblog ainsi que le petit dernier québécois Radioactif.tv. Dans ce dernier cas, voir : Le Soleil (01-02-2007). Le Québec a son YouTube. Jeudi, p. A6.

Parmi les innovations, un agrégateur de télévisions généralistes, service gratuit mais avec pub : Le Devoir (05-03-2007). Technologie. Joost, la télévision en ligne à la manière de Kazaa et de Skype, par Bruno Guglielminetti. Lundi, p. B7.

6 «La diffusion des quotidiens gratuits est en pleine explosion. Et la diffusion des quotidiens payants a reculé de 4,5\% en Amérique du Nord et de 6,3\% en Europe entre 2000 et 2005. ".

Le Devoir (24/25-02-2007). Médias. Quel avenir pour la presse écrite? Au-delà du scénario catastrophe, Internet et les nouveaux médias bouleversent le marché des journaux et des magazines, par Paul Cauchon. Samedi et dimanche, p. E 5.

Colloque: Radiodiffusion et nouveaux médias: modèles d'affaires et régulation, organisé par le Consortium canadien de recherche sur les médias et le Centre d'études sur les médias, à Montréal, 24 novembre 2006. Textes à paraître en 2007 dans les Actes du colloque. 


\section{François DEMERS}

elles, sont présentes non seulement sur les écrans vidéos, de cinéma et de télévision mais aussi de plus en plus sur les ordinateurs et les téléphones. Quant à la musique, elle passe partout. En résumé, les associations qui paraissaient si fortes entre des supports techniques, des modes de distribution, des formules de financement et des contenus, se distendent quand elles ne se rompent pas carrément.

Ainsi, les contenus médiatiques sont jaugés un à un sous l'angle de leur valeur marchande. Pour sa part, le professeur en économie des médias à la Jönköping University en Suède, Robert Picard a présenté à Montréal une échelle à trois barreaux servant à mesurer cette valeur. Au sommet, les contenus les plus attrayants, tels les films à succès et les sports. En dessous : les contenus qui génèrent un trafic suffisamment important pour attirer de la publicité ou d'autres formes de soutien financier. C'est la recette de Google, Yahoo, MyPlace, et YouTube. Enfin la catégorie des contenus de faible attrait, que l'on est forcé de donner dans des bouquets formés avec d'autres produits plus attrayants. Ce qui est le cas d'une bonne partie de l'information, comme mis en évidence par la vague des quotidiens gratuits et "la culture de la gratuité » qui paraît inhérente à l'Internet ${ }^{8}$.

Dans le remue-ménage des stratégies d'entreprise (Rabeau, 2006) et des expériences menées par les médias traditionnels (Bélanger, 2006), il y a cependant deux problèmes de fond qui tracent les limites du jeu possible. Le premier, c'est l'abondance des producteurs face à un nombre somme toute limité de consommateurs, d'où les efforts pour reconstruire les audiences de masse à travers les regroupements d'entreprises: YouTube avec Google, etc. Le second, c'est la position de faiblesse des producteurs de contenus dans le partage des dollars disponibles du côté des consommateurs. En effet, le journalisme s'inscrit au bout d'une filière technique qui alimente au moins cinq sortes d'entreprises. Au début de la chaîne, il y a les équipementiers : ils tirent leurs profits de la fabrication et la vente de téléviseurs, d'ordinateurs, d'appareils de téléphone (fixes et mobiles) et de baladeurs. Viennent ensuite les opérateurs de télécommunications et "transporteurs d'information " : câbles, téléphones, satellites. Suivent les producteurs des ponts entre les équipements et les contenus : les développeurs de la haute définition, des codeurs-décodeurs, des progiciels et logiciels divers. En quatrième lieu, les grossistes de contenus et services : les serveurs, ainsi que les médias traditionnels en partie. Enfin, tout au bout de la ligne, les producteurs de contenus, dont les chaînes traditionnelles

\footnotetext{
${ }^{8}$ La version 2007 du <http www.stateofthenewsmedia.com/2007> est centrée sur le défi de la gratuité.
} 


\section{Déstructuration et restructuration du journalisme}

de radio et de télé, les quotidiens, les magazines, les acteurs institutionnels et commerciaux qui opèrent des sites documentaires et promotionnels.

À cette extrémité de la chaîne, il ne reste qu'une faible tranche du budget du consommateur pour payer les contenus. En effet, une fois que le consommateur s'est procuré un ordinateur (plus une télévision, plus un téléphone, plus une caméra numérique, etc.), qu'il s'est abonné à un opérateur de télécommunications et à un serveur, il a déjà engagé l'essentiel de ses fonds. Comme par ailleurs, il a déjà été habitué par les quotidiens (largement subventionnés par la publicité), par les radios et télévisions traditionnelles, à un très faible prix ou à la « gratuité » des contenus, il se montre très réticent à verser une somme substantielle pour les contenus (par la tarification à l'usage, par l'abonnement, ...). . Dans ce contexte, il n'y a pas de surprise à voir fleurir sur Internet les entreprises en mesure d'offrir des contenus gratuits. Ainsi des sites de plus en plus nombreux émanent d'entreprises commerciales qui cherchent à rejoindre directement leurs clients actuels ou potentiels. D'un autre côté, de nombreux sites offrent des contenus déjà largement rentabilisés ailleurs : des films, des émissions de télé, des publicités et des informations utilisés sur d'autres supports (c'est la pratique dominante actuelle des médias traditionnels sur Internet). II y a aussi les agrégateurs de contenu qui « exploitent » (non sans résistance ${ }^{9}$ ) les produits du domaine public, comme le fait par exemple Google News. Plus à l'avant-garde encore, il y a ceux qui mettent à contribution les internautes eux-mêmes, tels YouTube ou MySpace, transférant sur eux la production du contenu, à la manière des « grandes surfaces » qui éliminent les employés en invitant les clients à explorer leurs entrepôts. Ces sites utilisent le réseautage social entre les usagers comme public de masse et greffent des publicités au trafic interactif entre les participants.

\subsection{Freinages et tâtonnements}

Mais, avant et avec le redéploiement et l'innovation, il y le freinage. Ainsi cette proposition en débat actuellement au Canada qui revendique, pour aider le

\footnotetext{
9 «Le géant des médias Viacom a porté plainte contre Google et sa filiale, en soulignant que 160,000 clips tirés de ses émissions avaient été téléchargés plus de 1,5 milliard de fois sur YouTube sans reversement de droits. "Les Échos (14-03-2007). Procès à 1 milliard de dollars contre YouTube et Google, par David Barroux. Mercredi, page 30.

Voir aussi : Le Devoir (23-03-2007). Une réponse à YouTube. NBC Universal et News Corp. Joignent leurs forces à AOL et MSN, par l'Associated Press. Vendredi, p. A7 Et: Le Soleil (07-04-2007). Google et L'Agence France-Presse signent un contrat de licence, PC et AP. Samedi, Affaires P. 47.
} 


\section{François DEMERS}

repli des télévisions généralistes, un paiement de droits à leur profit par les distributeurs du câble, des satellites et par Internet. En effet, les contenus des télévisions traditionnelles sont actuellement considérés comme du domaine public. L'une des façons d'obtenir le paiement de ces droits, proposent les télévisions traditionnelles, serait de mettre fin à la diffusion sur ondes hertziennes (les oreilles de lapin ${ }^{10}$ ), ce qui empêcherait carrément la saisie libre du signal émis «dans les airs " et forcerait les distributeurs à conclure des ententes avec les chaînes productrices. Parmi les autres mesures de transition, on peut ranger les tentatives des quotidiens traditionnels pour faire payer l'accès à leurs sites Internet d'information ou à certaines parties de ces sites. Compte tenu des coûts de production des contenus Internet essentiellement assumés par le journal traditionnel, et de la faiblesse des revenus publicitaires générés par les sites (5\% en moyenne), des médias tentent en effet de reconduire la formule de financement hors Internet, soit le mariage publicité / vente du journal (20\% des revenus actuellement). (PEJ, 2006, section Newspapers) II faudrait aussi documenter davantage la montée en force des quotidiens gratuits (Cordier, 2006), comme pis-aller. Au Canada, par exemple, Quebecor publie déjà un quotidien gratuit 24 heures à Montréal, face au Metro de ses concurrents Gesca et Transcontinental, et des 24 Hours à Montréal, Toronto et Vancouver. II a annoncé son intention d'en lancer dans d'autres villes au début de $2007^{11}$. Dans le cas de la France, le choc des quotidiens gratuits sur la presse payante prend l'allure d'un véritable séisme (Augey et al., 2005).

Un autre des mécanismes de freinage de la décroissance est l'intégration dans de plus grands groupes commerciaux (conglomérats, groupes et chaînes) afin d'y bénéficier de l'entraide de plusieurs entreprises-sœurs et d'y trouver des fonds pour supporter les déficits prolongés et faire les réinvestissements qui, espère-t-on, vont permettre des économies d'échelle et une expansion des revenus. Ainsi, dans le cadre du dernier congrès de la Fédération professionnelle des journalistes (FPJQ), le grand patron de Quebecor, PierreKarl Péladeau (PKP) s'est livré à une charge ouverte contre la pratique de l'étanchéité des salles de nouvelles des différents médias qu'il possède. Pour lui, l'étanchéité entre les salles de rédaction est « un concept de plus en plus obsolète et anachronique ». Celle-ci lui a notamment été imposée par le Conseil de la radiodiffusion et des télécommunications canadiennes (CRTC) quand son

\footnotetext{
${ }^{10}$ Aujourd'hui, il ne reste que $12 \%$ des Canadiens qui reçoivent la télévision par ce moyen. Le Devoir (16-10-2006). La fin des oreilles de lapin, par Paul Cauchon, Lundi, page B7.

${ }^{11}$ Le Devoir (27-11-2006), Congrès de la Fédération professionnelle des journalistes du Québec - Journaux gratuits et sites Web à l'agenda de Quebecor, par Paul Cauchon, lundi, p. B7.
} 


\section{Déstructuration et restructuration du journalisme}

groupe, Quebecor, s'est porté acquéreur du réseau privé de télévision TVA, le numéro 1 au Canada-français. Le CRTC lui a alors prescrit la mise sur pied d'un comité de surveillance qui établirait une séparation nette entre ses journaux et la chaîne de télévision. PKP plaide plutôt pour la convergence dont il s'est fait le promoteur dès 2003 au moment du lancement de Star Académie sur "sa » télé. De son côté, peu de temps avant le congrès de la FPJQ, le grand patron des services français de Radio-Canada (la chaîne publique, numéro 2 en matière d'audimat), Sylvain Lafrance avait indiqué la même orientation en faisant la promotion publique de ce qu'il nomme "l'intégration des services " et qui conduit à la circulation des journalistes entre la radio et la télévision ${ }^{12}$.

C'est la " convergence " organisationnelle, soutenue par la convergence économique et financière, elle-même ancrée sur la convergence numérique. Cette voie de solution est antérieure aux défis actuels puisque la tendance à la concentration et à la monopolisation de la propriété des médias est inhérente à la commercialisation, laquelle est la règle du jeu des médias depuis leurs débuts comme entreprises. C'est pourquoi la thématique de la concentration de la propriété des médias hante périodiquement les élites canadiennes ${ }^{13}$. Ainsi, à la fin des années 1960, au Québec, elle a été l'un des terrains de la lutte politique entre les indépendantistes québécois et les fédéralistes canadiens, ce qui par ailleurs a provoqué l'auto-structuration du groupe des journalistes de langue française en gestation depuis le début du siècle (Demers et Le Cam, 2004). Plus tard, au tournant du XXle siècle, la bulle spéculative des valeurs boursières technologiques a rendu possible un bond en avant de cette concentration (Demers, 2005). Celui-ci a donné lieu à des achats/ventes spectaculaires de médias, dont, aux États-Unis, l'emblématique AOL-Times-Warner . Au Canada, ces transactions géantes ont bouleversé le paysage entrepreneurial (Demers, 2000, 2003).

Une autre réaction des médias traditionnels à leur déclin, particulièrement remarquable à la Société d'État Radio-Canada, c'est le glissement du traitement sérieux des choses sérieuses vers les formules mises au point par les médias

\footnotetext{
12 Le Devoir (27-11-2006-2). Médias - La convergence pour sauver la presse, par Paul Cauchon. lundi, p. B7.

Voir aussi : Le Devoir (10-10-2006), Quebecor ne veut plus de frontières entre les salles de nouvelles, par Paul Cauchon, mardi, p. 1.

Et : Le Devoir (28-08-2007), Convergence maison à Radio-Canada, Par Paul Cauchon. Lundi, p. B6

${ }^{13}$ Le Soleil (14-04-2007), Le CRTC s'intéresse à la concentration des médias, par la presse canadienne, samedi, cahier Affaires, p. 47.
} 


\section{François DEMERS}

«populaires »: plus de couverture des thèmes légers, priorité aux contenus proches des conversations de la vie quotidienne, plus d'images, plus de gros titres, plus d'émotions, plus de potinage à propos des gens riches et célèbres, plus de cas individuels et de témoignages de quidams, plus de scénarios « interactionnels » pour les présentateurs, animateurs et reporters, d'ailleurs davantage "représentatifs» du public anticipé. Dans le contexte de la fragmentation des publics, le populaire est le lieu où il est encore possible de faire de l'audience (Demers, 2006).

Dernier exemple de stratégie de sortie de crise : les tentatives d'exploitation multimédia de contenus réalisés pour un seul support et, de manière plus générale, par le redéploiement des médias traditionnels vers l'Internet. Un rapport récent remis au gouvernement français propose même carrément que les quotidiens de ce pays s'orientent résolument et massivement dans cette direction ${ }^{14}$. Aux Etats-Unis, l'étude "The State of the News Media » démontre que cette orientation est déjà en voie de réalisation $(P E J, 2007)^{15}$. De tous côtés, la marche vers des ensembles différents est clairement en branle.

\section{L'hybridation des contenus}

Les contenus sont un deuxième niveau de destructuration / restructuration des ensembles constitutifs des médias traditionnels. La formule que ceux-ci avaient mise au point impliquait le mariage de trois grandes catégories de contenus : publicité, information journalistique et divertissement, d'abord dans la

\footnotetext{
${ }^{14}$ La presse au défi du numérique <http:// www.culture.gouv.fr/culture/actualites/>.

15 Stratégies (01-03-2007). Les stratégies Internet des news magazines. P. 30-33 $<$ http://www.strategies.fr> une publication de la division Presse du groupe Reed Business Information SAS. $\mathrm{N}^{0} 1446$, où l'on parle de "l'ère du bimédia ».

Le Monde (07-03-2007). Les quotidiens tirent profit de leurs sites Internet, par Pascale Santi, mercredi, p. 19

Le Devoir (07-06-2006). Le Times lance des actualités télévisées sur Internet, par l'AFP, mercredi, p. B7 :

"Le Times envisage de diffuser par la suite des images proposées par ses lecteurs «si elles sont intéressantes en terme d'actualité ».

Le Devoir (18-04-2006). La télé sur Internet : de plus en plus proche, par Paul Cauchon, mardi, p. B7 :

« $A B C$ fait le grand saut. La chaîne américaine, propriété du groupe Disney, a en effet annoncé la semaine dernière qu'elle proposera le mois prochain de regarder quatre de ses émissions directement sur son site Internet ».

Le Devoir (13-03-2007). Le Nouvel Observateur lance sa télé en ligne, par Le Devoir. Mardi, p. B 8

Infopresse (12-02-2007). Le New York Times sera web, par Vanessa Quintal. <http://www.infopresse.com/article.aspx?id=20782> site visité le 20-02-2007.
}

tic\&société - 1(1), 2007 


\section{Déstructuration et restructuration du journalisme}

presse écrite, puis à la radio, plus tard à la télévision (Bernier et al., 2005).) La recette réalisait la logique du tout-à-tous qui permettait de construire de larges audiences que l'on pouvait ensuite offrir aux annonceurs, les véritables sources de revenus de l'entreprise. Plus tard, dans un contexte de multiplication des médias et de leur spécialisation, une tendance à l'autonomisation de ces contenus les uns par rapport aux autres s'est dessiné.

Ainsi, les médias publicitaires se sont multipliés jusqu'à la formule des «consumer magazines» qui épousent les apparences des médias «indépendants » (Cavelier-Croissant et al., 2005) ou celle des «médias de sources » qui sont produits par des institutions et des entreprises, encore là avec les apparences de l'indépendance des médias traditionnels mais qui sont en fait un prolongement de leur publicité (SantAnna, 2006). Sur Internet, ces sites promotionnels se développent sous toutes sortes de formes, y compris extrêmes: "Destiné aux jeunes de 21 à 25 ans, Bud-TV (combinaison de chaînes télé et de réseau social conçu par la brasserie Anheuser-Busch) leur proposera des films courts, des émissions et, il va s'en dire, de la publicité, vraie et de la parodie. Accessible tout autant au Canada qu'aux États-Unis, Bud-TV permettra à ceux qui s'inscrivent (car il faut obligatoirement s'inscrire pour y pénétrer) de participer à des concours. ${ }^{16}$ Lors du colloque de Montréal, le consultant Ken Golstein de Communications Management Inc. à Winnipeg a pour sa part souligné le cas récent de la firme Land Rover qui a décidé de laisser tomber sa publicité à la télévision et de créer son propre canal sur l'Internet.

De son côté, le contenu divertissant, qui disposait déjà d'une base autonome dans l'édition et dans les salles de cinéma, et qui était devenu la locomotive principale pour la construction d'audiences de la télévision traditionnelle, a pu se déployer de manière exubérante avec les chaînes spécialisées sur le câble et les satellites, aujourd'hui sur les sites Internet. Encore là, le consultant Golstein avait de nombreux exemples à offrir. "On a parlé des films, a-t-il dit, on pourrait aussi parler des sports. Dans 10 ans au plus, il est probable qu'aucun sport ne sera plus diffusé par ce qu'on tient pour un média traditionnel. Tous les sports seront offerts au consommateur par les équipes ou les ligues, qui deviendront à leur tour des médias. C'est déjà le cas du réseau YES à New York. Et

${ }^{16}$ Blogue MédiaBiz (13-02-2007). Bud-TV: Le brasseur lance son réseau social, par Michel Dumais.

<http://mediabiz.branchez-vous.com/2007/02/budtv_le_brasseur_lance_son_rs.html> site visité le 16-02-2007. 


\section{François DEMERS}

remarquez que cela n'a pas à se produire partout pour avoir un effet sur les médias traditionnels parce que du moment que les Yankees de New York ont un débouché, l'économie de leur relation avec ESPN ou avec l'un des réseaux plus traditionnels change. Les enchères ne sont plus simplement entre ESPN et NBC, elles sont entre ESPN, NBC et combien nous pouvons réaliser si nous le faisons nous-mêmes. 》

Dans le même sens, le tout que formait l'information journalistique regroupant tous les domaines (rubriques, cahiers et thèmes) est graduellement dépecé et offert dans des publications spécialisées, des chaînes et des sites thématiques, en fonction de la valeur de chacun de ces thèmes sur le marché. Parmi les sujets les plus appétissants, il y a le sport bien entendu, le contenu "people » à propos des gens riches et célèbres, ainsi que les questions économiques et financières. II y aussi à un degré moindre toutes ces « informations de services » que les médias traditionnels offraient tantôt sous la forme d'espaces publicitaires (les petites annonces, les offres d'emploi, les annonces immobilières et les horaires de cinéma et de spectacle...) tantôt sous celle d'information «soft » formant un environnement positif pour les annonces payées. Un phénomène analogue s'est produit en matière d'information internationale. Ce sont les chaînes télévisuelles d'information continue qui se sont emparées de ce créneau, dans la foulée de la mondialisation des communications. II y a eu CNN avec son heure de gloire pendant la Guerre du Golfe, puis Al-Jazirah pendant l'invasion anglo-américaine de l'Afghanistan et Fox News en 2003 pendant l'offensive de la même coalition en Irak (Demers, 2007). Combinées aux possibilités offertes aux cybernautes par l'accès direct aux médias étrangers sur Internet, ces chaînes transfrontières qui se déploient par câbles et par satellites sur de larges aires linguistiques ont largement devancé les médias nationaux (traditionnels ou même d'information continue) dans l'offre de ce service.

Dans l'ensemble donc, les médias traditionnels, tels les supermarchés, voient leur terrain de jeu se rétrécir. D'un côté, les boutiques spécialisées, de l'autre, l'Internet où les clients se servent eux-mêmes, comme dans les magasins-entrepôts. L'examen de leur réaction première à l'augmentation de la concurrence montre qu'ils s'accrochent à la formule des trois ingrédients: publicité, information, divertissement. La recherche sur le terrain montre par contre que les pratiques signalant aux consommateurs les différences entre les 3 catégories de contenus, sont souvent abandonnées ou manipulées. Pendant plus d'un siècle, dans le giron des médias traditionnels, s'étaient développées des manières de faire visant à afficher la "nature » des contenus. Le contexte de la fin du siècle a poussé plutôt à expérimenter en jouant de ces conventions, à tester des « identités plurielles » et à « hybrider les genres » (Bernier, 2005). 
Déstructuration et restructuration du journalisme

Les contenus qui marient publicité et information, sans l'afficher vraiment, augmentent. D'autre part, le traitement de l'information emprunte les formes du divertissement.

\section{L'autonomisation des composantes du journalisme}

Si on centre maintenant le propos sur l'information journalistique, on peut discerner là encore un processus de dissociation des éléments qui la composent. Le graphique ci-après rappelle la formule du journalisme d'information qui a fait la fortune des entreprises de presse jusqu'à récemment. $\mathrm{Au}$ départ, les entreprises médiatiques étaient en position de se faire dépositaires d'un magistère journalistique - un lieu à partir duquel agir d'autorité - en s'ancrant dans la reconnaissance sociale de la liberté d'expression publique, traduite en liberté de presse, elle-même couplée à la liberté d'entreprise. Ce magistère s'était déployé dans trois directions.

En premier lieu, les médias ont peaufiné un contenu, les nouvelles, qui allait devenir emblématique sous le nom d'information et d'événements. Les médias ont exercé leur magistère en s'imposant comme définisseurs de l' "agenda " de ce qui est neuf aujourd'hui, de ce qui est important, de ce qu'il faut savoir pour les conversations de la journée. Les médias - et leurs journalistes -se sont faits définisseurs de l'actualité, ils se sont proclamés missionnaires de l'information autrement inaccessible aux simples citoyens.

Par ailleurs, les entreprises de presse ont rapidement atteint le stade du monopole (national) ou du quasi-monopole sur les marchés régionaux. Elles ont été perçues et se sont présentées elles-mêmes, à des fins notamment publicitaires, comme les incontournables " places publiques ». C'est d'ailleurs à partir du paysage de leurs monopoles que l'imaginaire d'un pouvoir énorme des médias sur les esprits s'est construit dans la recherche des années 1950. Encore aujourd'hui, quand ils discutent des médias, plusieurs observateurs et commentateurs pensent d'abord et avant tout aux médias généralistes et se réfèrent à la conception qui veut qu'ils aient mandat, chacun, de permettre la rencontre de toutes les différences dans l'espace public qu'ils forment. Le magistère médiatique s'est donc épanoui aussi en tant que "gatekeeper » de l'accès à la parole et à la visibilité publiques. La liberté d'expression individuelle ne va paraître se réaliser vraiment que dans l'information journalistique diffusée par les médias. C'est pourquoi une grande partie du vingtième siècle va offrir le spectacle de la lutte des "sources", plus spécifiquement des organisations et 


\section{François DEMERS}

entreprises aujourd'hui souvent regroupées sous le chapeau de "la société civile ", pour démocratiser les médias. L'enjeu sera clairement l'accès aux médias pour des acteurs de plus en plus nombreux et articulés, dans le cadre de la mise en place de la "démocratie des groupes d'intérêts". II s'agira d'obtenir des médias qu'ils se reconnaissent "médiatiseurs ", qu'ils fassent circuler les points de vue des uns et des autres, qu'ils se fassent volontairement des intermédiaires de l'expression publique des autres, dans des conditions de respect minimal. L'invention de "l'objectivité » des reporters sera l'une des réponses à cette pression.

Enfin, l'appropriation par les médias de l'expression publique des pensées et opinions va leur permettre de se poser aussi comme des acteurs sociaux partiaux et même partisans, parmi les autres. Invoquant la recherche de la vérité et du raisonnable, ils vont se donner le droit d'avoir des opinions qu'ils vont présenter comme "éclairées », comme «indépendantes » et guidées par la Raison, sinon par le Bien commun. Autrement dit, leur magistère va prendre une troisième forme en leur découpant un droit d'accès privilégié à l'expression publique de leur point de vue.

\subsection{Trois composantes de l'information journalistique}

L'actualité (le journalisme d'information)

- Les nouvelles

- Les événements

- Les faits

- L' « agenda-setting »

L'expression publique (la liberté d'expression)

- Liberté d'opinion

- Place publique médiatique

- Espace (s) public (s)

- La communication publique

- Le débat public

- Les « gatekeepers »

L'autorité éditoriale (la liberté de presse)

- L'explication

- La vérification

- La vérité

Le mouvement déstructuration / restructuration est aussi perceptible dans les relations entre ces trois composantes de l'information. D'une part, l'augmentation de la concurrence et la multiplication des médias spécialisés 


\section{Déstructuration et restructuration du journalisme}

poussent chaque média à affirmer sa personnalité, son autorité éditoriale, pour cibler "son » public. On voit donc de plus en plus les médias traditionnels abandonner leur discours d'indépendance, d'objectivité, d' "au-dessus de la mêlée », au profit du simple rappel de la concurrence et de leur liberté entrepreneuriale. La place publique médiatique n'est plus faite de quelques médias en situation de monopoles mais d'une multitude de médias. Alors, c'est cette multitude qui a mission d'assurer l'expression publique et non chacun des gros médias à lui seul. Ainsi, la liberté de presse et d'entreprise, devenue honteuse au temps des médias traditionnels que l'on voulait de service public, resurgit de manière comparable à celle du temps des feuilles polémiques de la presse d'opinion du XVIIle siècle. Cette évolution correspond bien par ailleurs aux possibilités offertes à tout un chacun par la miniaturisation et la simplification des infrastructures d'expression publique par les médias, notamment sur Internet. Quant à l'expression du vrai à partir d'un lieu d'autorité, elle fait de moins en moins sens dans une société qui n'espère plus l'arbitrage par la Raison ou par la science, mais plutôt par la majorité démocratique, en recomposition constante.

Dans ce contexte, l'autorité éditoriale (en anglais : authoritative point of view) du média est perçue comme une expression d'intérêt parmi d'autres. Les médias et les journalistes se rangent dès lors parmi l'ensemble des acteurs sociaux intéressés, en débat public pour faire valoir leurs points de vue propres et conclure des alliances avec d'autres pour obtenir une majorité en leur faveur. (Certains acteurs sociaux, dont des journalistes, invoquent encore parfois le "Bien public ».) Même la formule des médias de référence - ces médias qui font circuler l'information entre les élites d'un pays donné et qui informent les élites étrangères à propos de ce pays - tels le Globe and Mail au Canada et à plus petite échelle Le Devoir au Québec, peut être interprétée dans le sens de la défense /promotion d'intérêts quand on les considère comme des médias spécialisés.

Lors du colloque de Montréal (voir note 7), Robert Picard faisait remarquer d'autre part que nous vivons actuellement un cycle d'expansion des expressions publiques. "Nous avons créé une situation où plusieurs voix peuvent aujourd'hui être entendues à l'aide des nouvelles technologies, mais le fait est que la plupart ont très peu d'auditeurs. Il y a très peu d'endroits où vont un grand nombre de gens ou qui reçoivent beaucoup d'attention. » L'abondance des possibilités d'expression a l'effet paradoxal de libérer les anciens médias de leur mission (devoir de service public) d'offrir l'accès-à-la-visibilité-publique- 


\section{François DEMERS}

pour-tous (la numéro 2 dans le tableau), ce qui leur permet en même temps de répondre avec plus de liberté à un contexte commercial nouveau.

En Amérique du nord anglophone, le transfert de cette «mission " vers Internet, notamment par les blogues ${ }^{17}$, wikis et forums, et des sites d'échanges de vidéos tel YouTube, est même célébré comme une avancée de la démocratie. En effet, qui dit monopole et magistère, qui dit " gatekeeping ", dit pouvoir, mais dit aussi résistance à ce pouvoir, contournements et détournements. L'histoire particulière de la colonisation des Etats-Unis a inscrit dans la culture de ce pays une valorisation de la résistance aux "grandes » institutions, publiques ou privées et une invocation de la démocratie directe de la base (grassroots democracy) qui s'est constamment réinventée en misant souvent sur les nouvelles technologies. Ainsi, juste avant l'appel à la communication horizontale par Internet, et aujourd'hui l'émerveillement devant le « citizen journalism » des blogues et vidéos, il y avait eu l'expression contreculturelle par les radios et télévisions communautaires. Peu à peu, cette tradition de résistance aux grands médias fait actuellement le pont dans les recherches en communication et science politique, avec des éclairages provenant d'autres horizons, tels les " cultural studies » développés pour rendre compte des résistances des milieux populaires aux contenus médiatiques bourgeois et plus tard, à celles des femmes face au biais structurel machiste des médias (Neveu et Mattelart, 2003). En ce sens, le Réseau d'étude du journalisme $(\mathrm{REJ})^{18}$, auquel l'auteur de ce texte appartient, a entrepris depuis peu un cycle de recherches sous l'éclairage de la résistance "ordinaire " aux médias et au journalisme, des «braconnages » individuels, des déviances toujours renouvelées à la marge, célébrées par un auteur comme Michel de Certeau (De Certeau, 1980; Lafrance, 1993).

\subsection{Journalistes professionnels et journalisme citoyen}

Chose certaine, du côté des journalistes professionnels, l'expansion de lieux d'expression publique échappant à leur gatekeeping est nettement perçue comme une menace, aux États-Unis comme en Europe ${ }^{19}$.

Quant à l'actualité, la troisième composante de la recette information, elle s'autonomise elle aussi. Dans sa version dépouillée, l'actualité est le produit

\footnotetext{
17 « De 8 millions en mars 2005 à plus de 72 millions en mars 2007 ». AFP (05-04-2007), Les blogs gonflés à bloc. 09,26 hres.

${ }^{18}<$ http://www.surlejournalisme.com>.

19 Le Monde (06-03-2007), Le journalisme, quand Internet est roi ... Quoiqu'elle prétende, la profession n'a pas grand-chose à opposer au succès des blogs, par Hervé Brusini, mardi, Débats, p. 24.
} 


\section{Déstructuration et restructuration du journalisme}

central des quotidiens gratuits prêts-à-jeter, elle se déroule sur la page d'entrée des sites des médias traditionnels, elle fait office d'épine dorsale des contenus en boucle des télévisions nationales et transnationales d'information continue. Indirectement, elle nourrit aussi les grands agrégateurs de nouvelles sur Internet, les Google et Yahoo. Elle est même la matière première d'expériences de production de bulletins de nouvelles entièrement robotisés, y compris le présentateur $^{20}$. Ce déploiement multi-supports a aussi relancé les agences de presse en tant que grossistes de ce type de contenu. Bref, l'actualité se révèle une matière malléable que l'on peut extraire carrément du contexte des médias traditionnels pour l'introduire dans de nouveaux ensembles. Par contre, on se rend compte que l'actualité en général a peu de poids mercantile, même si certains de ses segments peuvent se vendre mieux, tels le sport et la finance. Sur le marché des biens de consommation, l'information reste handicapée. Historiquement, elle a été offerte dans des médias gratuits ou très peu coûteux, et, aujourd'hui, les environnements nouveaux valorisent cette gratuité. Elle ne peut donc se rédéployer qu'en se re-mariant avec d'autres contenus et en se fondant dans de nouveaux environnements.

Ou encore, comme l'apparition des blogues et de certains sites amateurs a pu laissé craindre ${ }^{21}$ ou espérer, sa production pourrait échapper des mains des professionnels, médias et journalistes, pour être contrôlée par les individus et les sources dans un mouvement toujours renouvelé de «spins » jaillissant de tous côtés sur Internet ${ }^{22}$. L'activité journalistique amateure, le «journalisme citoyen ", pourrait produire des nouvelles au sens habituel de contenu des Actualités. Pour l'instant, cette possibilité paraît pourtant ne se réaliser

\footnotetext{
${ }^{20}$ Le Devoir (02-04-2007-2), Un journal télévisé à la carte, écrit par une machine, Le Monde, lundi, p. B7.

${ }^{21}$ Le colloque organisé à Toronto le 5 avril 2007 par la Canadian Journalism Foundation et intitulé : The tech effect on news reporting - is traditional journalism dead? Reposait précisément sur le spectre du citoyen-reporter..

${ }_{22}$ Le 5 avril 2007, la Canadian Journalism Foundation a tenu à Toronto un colloque sur le thème : The tech effect on news reporting - is traditionnal journalism dead? Dans l'appel à participation, on pouvait lire : « The Internet has revolutionized the way we publish and consume information. "Citizen journalists"can immediately broadcast breaking news to millions of people through blogs and online communities, while audiences download free podcasts and webcasts specific to their niche interests. In effect, the world has come to expect personalized, 24/7 news coverage. What impact does this have on traditional media outlets, from both an editorial and business perspective? Can the news institutions of the past compete with today's new media mavericks? » Les «traditional media outlets " sont nettement au centre des préoccupations.
} 


\section{François DEMERS}

qu'exceptionnellement. Les blogues sont pour l'essentiel consacrés à l'expression du soi et au commentaire, parfois à propos de l'actualité ... fournie par les médias professionnels. II arrive aussi que certains contenus sur Internet font la nouvelle ... dans d'autres médias hors Internet.

II reste que l'initiative amateure est stimulée par les outils techniques dont les caméras et téléphones vidéos. Aussi, les professionnels sont-ils à mettre en place des mécanismes pour intégrer dans leurs routines des procédures de veille pour s'approprier ce matériel gratuit et assurer son exploitation commerciale. Yahoo et Reuters se sont entendu pour distribuer les photos des citoyens sur leurs sites, et dans le cas de Reuters, sur son fil de presse. Associated Press vient à son tour de faire une entente avec un portail citoyen canadien, NowPublic.com aux termes duquel l'agence AP distribuera en syndication des nouvelles produites par le portail civique dans ses fils de presse $^{23}$. De son côté, CNN a mis en place à son bureau-chef d'Atlanta un centre de production qui reçoit les vidéos amateur de partout, qui les évalue et les retravaille au besoin avant de les diffuser. En pratique, la plupart du temps, ces images sont liées à des soubresauts de la nature, des ouragans, des éboulements, etc. Fait à noter : " CNN does not pay i-Reporters. They must give CNN a non-exclusive license forever. In return, the citizen reporters get bragging rights ${ }^{24}$.

\section{Vedettariat et emplois atypiques}

Depuis l'invention du journalisme d'information, le sort des journalistes professionnels, en tant que travailleurs qui gagnent leur vie avec cette activité, est lié principalement à la production de l'actualité. Ils ne peuvent survivre ou prospérer qu'en suivant ses migrations. En tant que groupe plus ou moins autoorganisé par une histoire nationale, ils peuvent être profondément déstabilisés par les changements en cours. C'est notamment le cas des journalistes québécois dont les organisations ont grandi à l'ombre des médias traditionnels (Demers et Le Cam, 2006). Comme ces derniers sont encore les employeurs principaux, les organisations de journalistes sont directement touchées par leur repli. D'ailleurs, de manière générale dans les pays libéraux, la dislocation des éléments et leur recomposition peut se réaliser d'autant plus facilement que le corps professionnel des journalistes n'est défini ni par un «titre réservé », ni par

\footnotetext{
${ }^{23}<$ http://mediabiz.branchez-vous.com/2007/02/journalisme_civique_entente_de.html> ${ }^{24}$ Global Journalist (12-2006). Anyone Can Report for CNN. Citizen Reporters Need Only a Nose for News and the Right Equipment, by Joshua A. Loory. Reynolds Journalism Institute and Missouri School of Journalism <http://www.globaljournalis.org>, Vol. 12, nos $3 \& 4$, Pages $7 \& 8$.
} 


\section{Déstructuration et restructuration du journalisme}

des «actes réservés» inscrits dans une loi, comme le sont ceux des professions libérales. Des journalistes, auto-désignés comme tels, peuvent constamment surgir de partout; n'importe qui peut poser des gestes habituellement considérés comme journalistiques.

De manière plus globale, les défis relatifs à l'identité du groupe des journalistes, à son statut et à ses bénéfices ainsi que les apprentissages de pratiques nouvelles, s'inscrivent dans le contexte d'une réorganisation générale du travail caractérisée notamment par la montée des emplois « atypiques» $\left(\right.$ Bernier, 2003) ${ }^{25}$. Celle du travail des journalistes prend la forme d'une augmentation du nombre de ceux qui sont refoulés (!) dans le rôle modeste de rédacteurs (pupitreurs ou journalistes assis), lesquels se consacrent aux tâches de re-traitement du matériel tandis qu'émerge une classe de journalistes vedettes aspirés par l'industrie du spectacle ${ }^{26}$. Le livre Les nouveaux journalistes (Lapointe et Dumont, 2006) consacre son deuxième chapitre (pages 23-51) à ce qu'il intitule " Un métier (de plus en plus) précaire ». II prend acte de la réduction du bassin des emplois à temps plein, aux conditions de travail bien définies et offrant une possibilité de carrière dans le long terme, c'est-àdire pour l'essentiel les emplois syndiqués dans les grands médias traditionnels. II s'appuie par ailleurs sur diverses études, notamment la thèse de doctorat de Martine D'Amours (1993), elle-même ancienne journaliste pigiste, pour affirmer et prédire la croissance des emplois précaires (à temps partiel, sur appel, temporaire, à l'acte, à domicile) ${ }^{27}$. Ainsi, un grand nombre de professionnels, dont au premier chef les vedettes et les nouveaux entrants, doivent désormais fonctionner comme des entrepreneurs individuels. En tant qu'électrons libres sur le marché du travail, ils doivent savoir se recombiner constamment avec des ensembles divers et passagers.

Par ailleurs, dans de nouveaux médias et avec des moyens techniques plus divers, un grand nombre des journalistes professionnels sont mis au défi d'apprendre de nouvelles façons de produire l'actualité. Ainsi, les nouvelles

\footnotetext{
${ }^{25}$ Sans exclure même la délocalisation. Courrier International (02-2007), Les rédactions menacées de délocalisation, Diario de Milan, No 850, 15 au 18 février, p. 45.

26 «Mi-animateurs, mi-journalistes, pas tout à fait anonymes ni complètement vip, les chroniqueurs sont les chouchous des talk-shows à la française. " Ils mettent de l'avant une expertise patentée (l'information, l'humour, la philosophie, l'écriture, etc.) qui les distinguent des animateurs. TGV Magazine (03-2007). L'ère du Chroniqu'art, par Muriel Tramier. N 92, p. 20.

27 Les travailleurs dans cette situation sont souvent nommés «indépendants 》 (cette expression est utilisée par les services de la fiscalité canadienne) ou « autonomes».
} 


\section{François DEMERS}

technologies ont rendu possible la transmission vidéo en direct de leur travail sur le terrain : plusieurs doivent donc apprendre l'art de l'improvisation et de la conversation apparemment spontanée. D'un autre côté, la réorganisation de l'industrie médiatique les amène à travailler pour des employeurs qui offrent l'actualité sur plusieurs supports en même temps: par conséquent, certains journalistes doivent apprendre à maîtriser les traitements écrit, radio, vidéo et Internet du même contenu.

Nombreux sont ceux qui doivent se faire polyvalents et habiles dans le multitâches. Par exemple, López Vidales (2001), à partir de l'expérience espagnole, discute du profil du «journaliste digital » et en fait ressortir deux traits centraux : travaillant pour plusieurs employeurs et plusieurs plates-formes dans le cadre de relations d'emploi variables. II y a l'expérience menée au Washington Post où un "continuous desk news " coordonne la production simultanée pour la télé, la radio, l'écrit et l'Internet ${ }^{28}$. Mais l'un de ces profils expérimentaux est plus audacieux, c'est celui des «mojos » - mobile journalists - qui doivent produire des vidéo-clips en même temps que des segments-radio et des textes écrits à partir d'un poste de travail mobile et d'équipements légers ${ }^{29}$. Gannett, la chaîne de journaux qui publie le célèbre (et novateur en son temps) USA Today, a lancé une opération massive dans cette direction ${ }^{30}$. Au Québec, Canoë (Quebecor) a annoncé l'embauche de journalistes mobiles (mojos) qui diffuseront sur la Toile des clips d'informations quotidiennes.

De manière générale, ce sont tous les membres des nouvelles cohortes de journalistes qui doivent maîtriser un plus grand nombre de procédures

\footnotetext{
${ }^{28}$ Le Devoir (30-10-2006). Savoir tout faire, et plus encore, par Paul Cauchon. Lundi, p. B6.

29 « Un Mojo. Habituez-vous à ce terme, car vous l'entendrez régulièrement au cours des prochains mois. Et non, cela n'a rien à voir avec le charme suranné d'Austin Powers mais tout avec une nouvelle génération de journalistes, les journalistes mobiles, ceux qui feront tout autant leur recherche, leur tournage avec leur caméra vidéo et leur montage final. Bref, du TQS de première génération avec les outils modernes et adaptés. Lors de sa création, les premiers journalistes de TQS devaient eux-même tourner et réaliser leur reportage, sans caméramen ni preneurs de son. L'encombrement des outils technologiques alors disponibles a empêché ce projet d'être mené à terme. » Blogue MédiaBiz (02-02-2007). De la WebTélé pour Canoë: on engage des mojos, par Michel Dumais.

< http://mediabiz.branchez-vous.com/2007/02/de_la_webtl_pour_cano_on_engag.html> site visité le 16-02-2007.

${ }^{30}$ The Washington Post (04-12-2006), A Newspaper Chain Sees Its Future, And It's Online and Hyper-Local, by Frank Ahrens. Monday, p. A01.
}

tic\&société - 1(1), 2007 


\section{Déstructuration et restructuration du journalisme}

techniques ${ }^{31}$ et de formes discursives ${ }^{32}$ afin de pouvoir survivre sur un marché de l'emploi changeant et à reconquérir constamment. Dans leur cas, la compétence multimédias et la production de contenus adaptés à la circulation multisupports semblent définir les profils d'avenir. La déstructuration des emplois traditionnels de leurs aînés (pour un seul patron, pour un seul support) débouche sur des pratiques professionnelles quotidiennes restructurées autour de la polyvalence et du multitâches.

\section{Conclusion}

En somme, plus qu'il y a dix ans, les traits de la restructuration se dessinent à travers la déstructuration de l'ordre ancien, du moins quand on examine la situation comme il a été fait ici, sous les quatre angles des entreprises-médias, des combinaisons de contenus, des composantes de l'information journalistique et des pratiques des journalistes professionnels. Le passage du temps permet de répondre plus précisément à la question de départ posée dans le texte de 1996: «A-t-on atteint, comme le croient et le craignent plusieurs dans les milieux journalistiques, une masse critique de changements qui feraient que le journalisme est en train de se métamorphoser en quelque chose d'autre? » (Demers, 1996) L'analyse d'aujourd'hui montre en effet que «le journalisme » est resté le même, pour autant qu'on le ramène à l'activité : la production de nouvelles, qui a été considérée comme emblématique de son invention au XIXe

${ }^{31}$ Le Devoir (12-02-2007). L'AFP lance AFPTV International, par l'Agence FrancePresse. Lundi, p. B8. Une quarantaine de «journalistes vidéo 》 (en anglais: video journalists ou $\mathrm{VJ}$ ) sont utilisés pour ce service.

Voir aussi : Le Devoir (20-02-2007), Couverture de la campagne électorale - La presse canadienne à l'heure du clip vidéo sur Internet, par Paul Cauchon. Mardi, p. A3.

" La section anglophone de la Presse canadienne (Canadian Press) fournit déjà des vidéos depuis quelques mois à ses clients, mais c'est la première fois que la section francophone s'y met. Et c'est la première fois que l'on tente une telle expérience dans une campagne électorale canadienne. [ ... ] Reuters et Associated Presse (AP) fournissent déjà une couverture vidéo de différents événements (La Presse canadienne vend d'ailleurs les produits d'AP au Canada). ".

Ou encore : Le Devoir (10/11-02-2007), Journaliste ou caméraman?, par Paul Cauchon, samedi et dimanche, p. E4.

${ }^{32}$ Par exemple : apprendre comment écrire des articles qui seront bien référencés par Google.

Blogue Mediabiz (09-02-2007). Journaux en ligne: écrire pour les lecteurs ou Google?, par Michel Dumais.

<http://mediabiz.branchez-vous.com/2007/02/journaux_en_ligne_crire_pour_l.html> site visité le 16-02-2007. 


\section{François DEMERS}

siècle (Chalaby, 1998). Pour le reste de ce qui est souvent inclus dans ce qu'on désigne par le terme: l'expression publique d'opinions, le prestige d'une " profession », les entreprises de presse, ..., tout cela se recompose en un ensemble différent de l'ordre construit autour des médias généralistes. C'est l'approche par la déstructuration qui fait apparaître cette continuité dans le changement général.

Quant aux journalistes professionnels, ils fonctionnent déjà dans cette nouvelle configuration.

"La perspective adoptée, pouvait-on lire dans l'introduction de 1996, s'adosse à l'hypothèse générale suivante: sur plus d'un siècle, les journalistes se sont construits une identité professionnelle dont les principaux paramètres subissent une crise aiguë de "destruction créatrice" (Schumpeter, 1984:117) depuis le tournant des années 80, c'est-à-dire en synchronisme avec les crises - l'économique et l'idéologique - centrées sur l'Etat-providence. Autrement dit, pendant une période de calme relatif qui aura duré près d'un siècle, l'industrie de l'information a assimilé l'onde de choc des novations nombreuses de la deuxième moitié du XIXe siècle. Autour des nouvelles machines, des nouveaux procédés de production et de vente se sont lentement cristallisés des organisations, des images de soi, des valeurs, des idéaux, des normes et des représentations du monde. L'identité d'un corps professionnel, les journalistes, a émergé et s'est structurée, source de prestige, de respect, d'autorité et de revenus pour ses membres (de Bonville, 1988). ”

Le portrait de l'état des choses, présenté ici, demeure à l'intérieur de l'hypothèse d'une crise de "destruction créatrice » du journalisme, laquelle se produit dans une société globalement en rupture/relance. Le changement technique, plus spécifiquement la filière de l'informatisation, est l'un des ingrédients actifs de ce moment de métamorphose. 


\section{Références bibliographiques}

ATTALLAH, P., 1989, Théories de la communication: Histoire, contexte, pouvoir, Montréal, Presses de l'université du Québec et Télé-Université.

AUGEY, D., M.-Ch. LIPANI-VAISSADE, D. RUELLAN et J.-M. UTARD, 2005, «Dis à qui tu te donnes... La presse quotidienne gratuite ou le marketing du don », dans RINGOOT, R. et J.-M. UTARD (dir), Le journalisme en invention. Nouvelles pratiques, nouveaux acteurs, Rennes, Presses Universitaires de Rennes (PUR), pp. 89-123.

BÉLANGER, P.-C., 2006, «Le déploiement du Web 2.0 et des supports mobiles: vers une transmutation des 'nouveaux' médias traditionnels », dans les actes du colloque Radiodiffusion et nouveaux médias : modèles d'affaires et régulation, organisé par le Consortium canadien de recherche sur les médias et le Centre d'études sur les médias, à Montréal, 24 novembre 2006. Texte à paraître en 2007.

BERNIER, J. et al, 2003, « Les besoins de protection sociale des personnes en situation de travail non traditionnelle ». Québec, 807 pages. En ligne à : http://www.travail.gouv.qc.ca/actualite/travail_non_traditionnel/Bernier2003/

BERNIER, M.-F., F. DEMERS, A. LAVIGNE, Ch. MOUMOUNI et T. WATINE, 2005. Pratiques novatrices en communication publique, Journalisme, relations publiques et publicité, Québec, Les Presses de l'Université Laval.

CAVELIER-CROISSANT V., W. SPANO, A. TOUBOUL et F. REBILLARD, 2005, "De la culture comme image de marque aux marques journalistiques de la culture - Epok, le consumer magazine de la FNAC ». dans Ringoot, R. et Utard, J.-M. (dir.). Le journalisme en invention. Nouvelles pratiques, nouveaux acteurs. Rennes, Presses Universitaires, collection Res Publica.

CHALABY, J. K., 1998, The Invention of Journalism, Great Britain, MacMillan Press Ltd..

CORDIER, É., 2006, «Metro International, la bible de la presse quotidienne gratuite », Les Cahiers du Journalisme, École supérieure de journalisme de Lille (ESJ) et Département d'information et de communication (DIC), no 16, automne, pp. 274-293.

D'AMOURS, M., 2003, Le travail indépendant: Un révélateur des mutations du travail. Montréal, Presses universitaires du Québec. Tiré de sa thèse de doctorat <http://www.irec.net/publications/459.pdf>. 


\section{François DEMERS}

DE BONVILLE, J., 1988, La presse québécoise de 1884 à 1914 - Genèse d'un média de masse, Québec, Les Presses de l'Université Laval.

DE CERTEAU, M., 1980, L'invention du quotidien - Arts de faire, Paris, Union générale d'éditions, collection 10 / 18.

DEMERS, F., 2007, « Les conditions d'une contribution du journalisme et des médias mondiaux au dialogue. L'arrivée d'Al-Jazira au Canada », Chapitre de livre à paraître dans les Actes du Séminaire international du GEPANC: Le dialogue des civilisations. L'Islam et l'Occident, sous les auspices de I'UNESCO, Université Laval, 6-7-8 mai 2005.

DEMERS, F. et F. LE CAM, 2006, « The Fundamental Role Played by Unionism in the Self-Structuring of Professional Journalists from Quebec », The Canadian Journal of Communication, Volume 31, number 3, pp. 659-674.

DEMERS, F., 2006, «Concentration de la propriété des médias et réaménagement de la place publique médiatique; leçons des affaires Voilà et Star Académie ", Les Cahiers du Journalisme, École supérieure de journalisme de Lille (ESJ), France, et Département d'information et de communication (DIC), Université Laval, Québec. No 16, automne, pp. 46-69.

DEMERS, F., 2005, « La convergence comme nouvelle pratique journalistique » dans BERNIER, M.-F., F. DEMERS, A. LAVIGNE, Ch. MOUMOUNI et T. WATINE, Pratiques novatrices en communication publique: Journalisme, relations publiques et publicité. Québec, Les Presses de I'Université Laval, pp. 77-101.

DEMERS, F. et F. LE CAM, 2004, «L'identité professionnelle des journalistes québécois Petite histoire d'un événement-repère ", Actes du Colloque interdisciplinaire Identités, Poitiers, les 23, 24 et 25 janvier 2002, pp. 157170

DEMERS, F., 2003, « Journalistes au Canada et au Québec : reconfiguration générale », Hermès, Paris, CNRS Éditions, no 35, pp. 165-173

DEMERS, F., 2000, "Concentration des entreprises de presse : vers une reconfiguration du paysage médiatique au Québec et au Canada ", Les Cahiers du Journalisme, École supérieure de journalisme de Lille (ESJ) et Département d'information et de communication (DIC), no 8, décembre, pp. 192-203

DEMERS, F., 1996, « Impacts des nouvelles technologies de l'information et de la communication (NTIC): Déstructuration (et restructuration?) du 
Déstructuration et restructuration du journalisme

journalisme ", Technologies de l'information et Société, Vol. 8. No 1, pp. 5570.

ELIAS, N., 1981, Qu'est-ce que la sociologie ?, Paris, Pandora éditions.

LAFRANCE, J.-P, 1993, La construction du public à la télévision. les nouveaux courants de pensée en télévision, Communication, vol. 14, no 2, pp. 171195.

LAPOINTE, P. et Ch. DUPONT, 2006, Les nouveaux journalistes. Le guide : Entre précarité et indépendance, Québec, Les Presses de l'Université Laval.

LÓPEZ VIDALES, N., 2001, "El impacto de las nuevas technologías de la comunicación en el periodista/comunicador del tercer milenio ", Comunicación y Sociedad, DECS, Universidad de Guadalajara, México. Núm. 40, julio - deciembre. pp. 183-198.

NEVEU, É. et A. MATTELART, 2003, Introduction aux Cultural Studies, Paris, Éditions La Découverte.

PEJ (Project for Excellence in Journalism) 2006 et 2007, The State of the News Media. School of Journalism of the Columbia University. $\ll$ http www.stateofthenewsmedia.com/2007 >>

RABEAU, Y., 2006, " La radiodiffusion en pleine transformation : la technologie, les consommateurs et les stratégies des entreprises ». Actes du Colloque : Radiodiffusion et nouveaux médias: modèles d'affaires et régulation, organisé par le Consortium canadien de recherche sur les médias et le Centre d'études sur les médias, à Montréal, 24 novembre 2006. Texte à paraître en 2007.

RUELLAN, D., 1993, Le professionnalisme du flou, identité et savoir-faire des journalistes français, Rennes, Presses Universitaires de Rennes (PUR).

SANTANNA, F., 2006, "Le journalisme brésilien : médias de source et profil du marché du travail ", Les Cahiers du Journalisme, École supérieure de journalisme de Lille (ESJ) et Département d'information et de communication (DIC), no 16, automne, pages 168-188.

SCHUMPETER, J., 1984, Capitalisme, socialisme et démocratie, Paris, Payot. 


\section{François DEMERS}

Pour citer cet article :

DEMERS F., «Déstructuration et restructuration du journalisme», Revue tic\&société [En ligne], Volume 1, n9, 2007, mis en ligne le : 15/02/2008, URL :

<http://revues.mshparisnord.org/lodel/ticsociete/index.php?id=298.>. 